\title{
Identification of cardiomyopathy associated circulating miRNA biomarkers in patients with muscular dystrophy using a complementary cardiovascular magnetic resonance and plasma profiling approach
}

Svetlana Becker ${ }^{1 \dagger}$, Anca Florian ${ }^{2 \dagger}$, Alexandru Patrascu ${ }^{3}$, Sabine Rösch ${ }^{3}$, Johannes Waltenberger ${ }^{2}$, Udo Sechtem $^{3}$, Matthias Schwab ${ }^{1,4,5}$, Elke Schaeffeler ${ }^{1 \dagger}$ and Ali Yilmaz ${ }^{2^{*}+}$

\begin{abstract}
Background: Duchenne and Becker muscular dystrophy (DMD and BMD) are X-chromosomal recessive neuromuscular disorders that are caused by mutations in the dystrophin gene and characterized by cardiac involvement. Circulating microRNAs (miRNAs) have been proposed as diagnostic biomarkers for various cardiovascular diseases. However, circulating miRNAs reflecting the presence and/or disease severity of cardiac involvement in DMD/BMD patients have not been described so far.

Methods: Sixty-three male patients with known MD and 26 age-matched healthy male controls were prospectively enrolled. All MD patients and controls underwent comprehensive cardiovascular magnetic resonance (CMR) studies as well as venous blood sampling on the same day.

Results: An impaired left ventricular (LV) systolic function (defined as LV-EF <55 \%) was detected in 29 (46\%) and presence of late gadolinium enhancement (LGE) indicative of myocardial fibrosis in 48 (76\%) MD patients with an exclusively non-ischemic pattern. Whereas no significant differences were observed for the 27 selected circulating miRNAs in MD patients with abnormal CMR findings (comprising structural and/or functional impairments) compared to those with completely normal CMR studies, a significant up-regulation of three miRNAs was observed in LGEpositive MD patients compared to LGE-negative ones: miR-222 (1.8-fold, $p=0.035)$, miR-26a (2.1-fold, $p=0.03)$ and miR-378a-5p (2.4-fold, $p=0.026$ ). A signature of these three miRNAs (miR-26a, miR-222 and miR-378a-5p) resulted in an area under the curve (AUC) value of 0.74 for the diagnosis of LGE-positive MD patients. In a multivariable model, three independent predictors for LGE presence were identified comprising not only clinical and laboratory markers (LV-EF: OR 0.47, $95 \%$ Cl 0.24-0.89, $p=0.021$ and elevated hs-Trop: OR 2559, $95 \% \mathrm{Cl}$ 2.97-22.04*1 $0^{5}, p=0.023$ ) but also the circulating miR-222 (OR 938, $95 \% \mathrm{Cl} 938.46,3.56-24.73^{*} 10^{4}, p=0.016$ ).

Conclusions: Up-regulation of circulating miRNAs miR-222, miR-26a and miR-378a-5p indicates the presence of myocardial scars in MD patients. Plasma miR-222 appears to be a promising novel biomarker reflecting structural - but not functional - cardiac alterations in MD patients.
\end{abstract}

Keywords: miRNA, Cardiomyopathy, Muscular dystrophy, Cardiovascular magnetic resonance, Late gadolinium enhancement

\footnotetext{
*Correspondence: ali.yilmaz@ukmuenster.de

${ }^{\dagger}$ Equal contributors

${ }^{2}$ Department of Cardiovascular Medicine, University Hospital Münster,

Albert-Schweitzer-Campus 1, building A1, 48149 Münster, Germany

Full list of author information is available at the end of the article
} 


\section{Background}

Duchenne and Becker muscular dystrophy (DMD and $\mathrm{BMD}$ ) are X-chromosomal recessive neuromuscular disorders that are caused by mutations in the dystrophin gene that subsequently lead to either total absence or structural impairment of the dystrophin protein. Since dystrophin is a central protein in the cell membrane of skeletal as well as cardiac muscle cells, MD patients do not only suffer from skeletal muscle weakness and wasting but also from progressive cardiomyopathy $[1,2]$. Since therapeutic treatment options for respiratory failure have tremendously improved within the last years, cardiac disease with a characteristic, non-ischemic pattern of left ventricular (LV) myocardial fibrosis leading to non-ischemic dilated cardiomyopathy, heart failure symptoms and ventricular arrhythmias has become a major cause of morbidity and mortality in MD patients [1, 3-5].

Within the last years, cardiovascular magnetic resonance (CMR) has evolved as an excellent tool for the early and sensitive diagnosis of cardiac involvement in MD patients, since this imaging modality does not only allow an accurate functional assessment of the human heart - but also enables non-invasive myocardial fibrosis detection based on techniques such as late gadolinium enhancement (LGE)-imaging or T1-mapping [6-8]. However, previous CMR-based studies in MD patients have also suggested that cardiac serum markers such as troponin or brainnatriuretic peptide (BNP) that are helpful to diagnose and monitor cardiac disease in many ischemic as well as nonischemic cardiomyopathies, are of limited clinical value in MD patients [9, 10]. Hence, the identification of novel serum biomarkers for diagnosis and monitoring of cardiomyopathy in MD patients (e.g. as a gatekeeper for subsequent CMR studies) is a clinically important, however, still unsolved challenge.

Recent advances in molecular diagnostics have opened novel avenues in defining the molecular basis and underlying pathophysiology of inherited diseases. In particular, microRNAs (miRNAs) have been identified as a novel class of both biomarkers and targets for therapy [11]. miRNAs are small (19-25 nucleotides), non-coding molecules and can modify gene expression by regulating mRNA stability or translation and thereby modify essential cellular functions [12]. Recent studies have shown that miRNAs are not only located inside cells (where they exert their function) but can also be found in different fluids such as blood [13]. Moreover, there is a growing body of evidence that circulating miRNAs can be used as diagnostic as well as prognostic biomarkers for different cardiovascular diseases $[14,15]$. However, circulating miRNAs reflecting the presence and/or disease severity of cardiac involvement in DMD/BMD patients have not been described so far.

Hence, the aim of the current study was to identify specific circulating miRNAs in the plasma of DMD/BMD patients that would allow a non-invasive and accurate diagnosis of cardiac disease in these patients.

\section{Methods \\ Study population}

As part of an ongoing prospective study, 63 male patients with known MD were prospectively enrolled between 2008 and 2015. A diagnosis of DMD $(N=12)$ or BMD $(N=51)$ has previously been made in specialized neurology centers based on clinical data, skeletal muscle pathology with dystrophin analyses and/or genetic testing $[10,16-18]$. The clinical degree of skeletal myopathy was clinically assessed as follows: $0=$ no clinical signs of myopathy; 1 = able to walk, unable to run; $2=$ unable to walk; 3 = unable to use hands.

In addition, 26 age-matched healthy male controls were enrolled between 2011 and 2014 and represented the control group. All MD patients and controls underwent comprehensive CMR studies as well as venous blood sampling on the same day. The study protocol complies with the Declaration of Helsinki and was approved by the local ethics committee (Ethik-Kommission Landesärztekammer Baden-Württemberg, Stuttgart, Germany). Informed consent was obtained from the patients prior to study inclusion.

\section{Blood sampling in MD patients and controls}

EDTA blood samples were collected on the same day of the CMR study. EDTA plasma was harvested by centrifugation of EDTA Blood Collection Tubes (Sarstedt, Germany) for $10 \mathrm{~min}$ at $4000 \mathrm{rpm}$. Aliquots of plasma supernatant were stored in cryotubes (Sarstedt, Germany) at $-20{ }^{\circ} \mathrm{C}$ until use. Both in MD patients and controls, laboratory determinations for cardiac biomarkers - high sensitive troponin I (hs-Trop) and brain natriureticpeptide (NT-proBNP) - were performed using standard methods and considered elevated when serum levels exceeded the upper laboratory reference limit. In addition, creatine kinase $(\mathrm{CK})$ levels were determined.

\section{miRNA extraction and quantification}

RNA was extracted from $400 \mu \mathrm{l}$ of plasma using mirVana miRNA isolation kit (Life technologies, USA) following the manufacturer's protocol, eluted in $75 \mu$ l elution solution and stored at $-80{ }^{\circ} \mathrm{C}$.

miRNA selection for quantification was based on literature data related to either cardiovascular diseases and/or DMD/BMD (Additional file 1: Table S1) were reverse transcribed using TaqMan MicroRNA Reverse Transcription Kit (Life technologies, USA). Individual stem-loop reverse transcription primers included in the predeveloped TaqMan miRNA assay (Life technologies, USA) were pooled at a final dilution of $0.05 \mathrm{x}$ for each primer. The final RT reaction volume of $7.5 \mu \mathrm{l}$ contained $0.15 \mu \mathrm{l}$ 
$100 \mathrm{mM}$ dNTP, $1.5 \mu \mathrm{l}$ multiscribe reverse transcriptase $(50 \mathrm{U} / \mu \mathrm{l}), 0.75 \mu \mathrm{l} 10 \times \mathrm{RT}$ buffer, $0.095 \mu \mathrm{l}$ RNase inhibitor $(20 \mathrm{U} / \mu \mathrm{l}), 3 \mu \mathrm{l}$ primer pool and $2 \mu \mathrm{l}$ of total RNA. The reaction was performed following conditions of manufacturer.

To improve sensitivity of miRNA quantification, a preamplification reaction was performed. TaqMan miRNA assays included in the TaqMan miRNA assay (Life technologies, USA) were pooled at a final dilution of $0.2 x$ for each assay. Pre-amplification reaction was done at $10 \mu \mathrm{l}$ final volume containing $5 \mu \mathrm{l}$ TaqMan PreAmp Master Mix (2X), $1.5 \mu \mathrm{l}$ of assay pool, $2.5 \mu \mathrm{l}$ of nuclease-free water and $1 \mu \mathrm{l}$ of cDNA. The pre-amplification PCR was run according to the manufacturer's protocol, the preamplification PCR product was diluted 1:5 with suspension buffer (Teknova AS, Norway) and stored at $-20{ }^{\circ} \mathrm{C}$ until need.

The miRNA expression levels were quantified by realtime PCR using TaqMan ${ }^{\circ}$ Universal Master Mix II (no UNG) and TaqMan miRNA assays (Life technologies, USA) on a real-time PCR BioMark system (Fluidigm Corporation, USA) following the manufacturer's protocol. Relative levels of miRNA expression were calculated by normalization to expression levels of miR-16 and thereafter multiplied by $10^{3}$ in order to increase readability in the respective tables. The following miRNAs had to be excluded from final analysis due to failing measurements: miR-1, miR-31, miR-34c, miR-95, miR-133a, miR-208a, miR-208b, miR-499a-3p, miR-499a-5p and miR-539.

\section{CMR data acquisition}

ECG-gated CMR studies were performed on a 1.5-T scanner (Aera, Siemens Medical Solutions, Erlangen, Germany) using commercially available cardiac software, electrocardiographic triggering, and cardiac-dedicated surface coils. Cine-imaging was performed using a steady-state-free-precession (SSFP) sequence in three long-axis slices (four-, three- and two-chamber) and a stack of short-axis slices completely covering the LV. LGE-imaging was performed using a T1-weighted inversion recovery gradient-echo sequence 10-15 min after intravenous contrast administration $\left(0.15 \mathrm{mmol} / \mathrm{kg}\right.$ Magnevist $\left.{ }^{\circ}\right)$ in the same imaging planes as the cine-images.

\section{CMR data analysis}

CMR analysis was performed off-line by two experienced readers blinded to gender and clinical characteristics. Ventricular volumes, ejection fraction and LV mass were derived by contouring endo- and epicardial borders on the short-axis cine images and indexed to body surface area. LGE presence and pattern were first visually assessed on the short-and long-axis images by using the 16-segment AHA model [16]. Second, LGE extent was planimetered on the short-axis contrast images with the use of ImageJ software (National Institutes of Health, Bethesda, Md, USA) and an image intensity level $\geq 3$ SD above the mean of remote myocardium was used to define LGE indicative of damaged myocardium as described previously (National Institutes of Health, Bethesda, Md, USA) and expressed as percentage of total LV mass [19].

An abnormal CMR was defined by at least one of the following findings: i) LV ejection fraction (LV-EF) less than $55 \%$, ii) RV ejection fraction (RV-EF) less than $45 \%$, iii) presence of LGE in at least one myocardial segment (AHA segmentation), and was considered as sign of cardiac involvement.

\section{Statistical analysis}

Continuous variables are expressed as mean \pm SD. Skewed variables are expressed as median and interquartile range (IQR). Categorical variables are expressed as frequency with percentage. $t$-Student test was used for comparison of normally distributed variables, while Mann-Whitney $U$ test was used for comparison of non-normally distributed variables. Non-parametric Kruskal-Wallis test with Bonferroni post-hoc correction was used in case of multiple comparisons of non-normally distributed variables. The Chi-square test with Yate's correction was used to compare non-continuous variables expressed as proportions. Parametric Pearson or non-parametric Spearman correlations were used as corresponded for correlation analysis. In order to find independent predictors for abnormal CMR findings, i.e. LGE presence, a univariable regression analysis was first performed. Second, the parameters with significant $\mathrm{p}$-values were introduced into the multivariable regression analysis. Extremely skewed miRNAs (skewness statistic $<-2$ or $>2$ ) were Log10 transformed before introduced in the regression analysis. Finally, receiver operating characteristic curves (ROC) were analyzed to assess specificity and sensitivity of single plasma miRNAs as well as their combination using multiple logistic regression analysis. Statistical analysis was performed using SPSS software for Windows (version 20, SPSS, Chicago Illinois, US). A $p$-value $\leq 0.05$ was considered statistically significant.

\section{Results}

\section{Patient characteristics}

The study group consisted of 63 male MD patients comprising 12 (19\%) patients with DMD and 51 (81\%) with BMD with a median age of $31 \pm 15$ yrs (Table 1 ). DMD patients were younger compared to BMD ones ( $18 \pm 5$ yrs vs. $33 \pm 15$ yrs, $p<0.0001)$. The control group comprised 26 healthy male volunteers aged $36 \pm 13$ yrs $(p=0.09)$. There was no patient with a history of coronary artery disease (CAD) and/or ischemic cardiomyopathy and/or valvular disease. The respective cardiovascular risk profile is illustrated in Table 1. Serum measurements revealed elevated 
Table 1 Patient characteristics

\begin{tabular}{lllc}
\hline & MD & Controls & $p$ value \\
& $N=63$ & $N=26$ & \\
\hline Male, $n(\%)$ & $63(100)$ & $26(100)$ & 1.00 \\
Age, years & $31 \pm 15$ & $36 \pm 13$ & 0.09 \\
BMl, kg/m ${ }^{2}$ & $24 \pm 4$ & $25 \pm 3$ & 0.36 \\
Skeletal muscle status & $2(3) / 43(68) /$ & $26(100) / 0(0) /$ & $<0.0001$ \\
0/1/2/3, $n(\%)$ & $13(21) / 5(8)$ & $0(0) / 0(0)$ & \\
Hypertension, $n(\%)$ & $3(5)$ & $0(0)$ & 0.55 \\
Diabetes, $n(\%)$ & $0(0)$ & $0(0)$ & 1.00 \\
ACE inhibitor, $n(\%)$ & $16(25)$ & $0(0)$ & $\mathbf{0 . 0 0 5}$ \\
Beta-blocker, $n(\%)$ & $14(22)$ & $0(0)$ & $\mathbf{0 . 0 0 8}$ \\
CK, U/L & $1226(653-2424)$ & $120(99-169)$ & $<\mathbf{0 . 0 0 0 1}$ \\
Elevated hs-Trop, $n(\%)$ & $30(48)$ & $0(0)$ & $<\mathbf{0 . 0 0 0 1}$ \\
Elevated NT-proBNP, $n(\%)$ & $3(5)$ & $0(0)$ & 0.55 \\
\hline Bold & & &
\end{tabular}

Bold text indicates a significant $p$-value of $<0.05$

hs-Trop values in 30 (48 \%) MD patients $(p<0.0001$ vs. controls) whereas NT-proBNP levels were increased in 3 (5\%) MD patients only ( $p=0.55$ vs. controls).

\section{Major CMR findings}

The detailed results of the CMR studies performed in MD patients and controls are given in Table 2. An impaired LV systolic function (defined as LV-EF $<55 \%$ ) was detected in 29 (46\%) MD patients ( $p<0.0001$ vs. controls) and a reduced RV systolic function (defined as RV-EF $<45 \%$ ) in 10 (16\%) MD patients ( $p=0.031$ vs. controls). Presence of LGE indicative of myocardial fibrosis was detected in 48 (76\%) MD patients ( $p<0.0001$ vs. controls) with a median LGE extent of $10 \%$ (IQR $5 \%-24 \%$ ) of LV mass and an exclusively non-ischemic pattern (Fig. 1a-b). There was a strong negative correlation between LGE extent and LV-EF (Spearman's rho $=-0.753, p<0.0001$ ). Altogether, any pathological CMR finding was observed in

Table 2 Overview CMR results

\begin{tabular}{lllc}
\hline & MD & Controls & $p$ value \\
& $N=63$ & $N=26$ & \\
\hline LV-EDVi, ml/m² & $85 \pm 30$ & $86 \pm 16$ & 0.80 \\
LV-ESVi, ml/m² & $41 \pm 21$ & $31 \pm 9$ & $\mathbf{0 . 0 0 3}$ \\
LV-massi, g/m² & $60 \pm 16$ & $58 \pm 10$ & 0.69 \\
LV-EF, \% & $53 \pm 12$ & $64 \pm 6$ & $<\mathbf{0 . 0 0 0 1}$ \\
RV-EF, \% & $53 \pm 9$ & $55 \pm 8$ & 0.47 \\
LV-EF <55 \%, $n(\%)$ & $29(46)$ & $0(0)$ & $<\mathbf{0 . 0 0 0 1}$ \\
RV-EF $<45 \%, n(\%)$ & $10(16)$ & $0(0)$ & $\mathbf{0 . 0 3 1}$ \\
LGE presence, $n(\%)$ & $48(76)$ & $0(0)$ & $<\mathbf{0 . 0 0 0 1}$ \\
LGE extent, \% & $7(1-17)$ & - & - \\
Abnormal CMR, $n(\%)$ & $49(78)$ & $0(0)$ & $<\mathbf{0 . 0 0 0 1}$ \\
\hline
\end{tabular}

Bold text indicates a significant $p$-value of $<0.05$
$49(78 \%)$ MD patients whereas all subjects in the control group demonstrated normal CMR findings $(p<0.0001)$.

No significant differences were seen between DMD and BMD neither in frequencies of $\mathrm{LV}$ and/or RV systolic impairment (42\% vs. $47 \%, p=1.00$ for LV; $25 \%$ vs. $14 \%$, $p=0.39$ for RV) nor in LGE presence (92\% vs. $73 \%$, $p=0.26$ ) or LGE extent. Consequently, there was also no significant difference in the prevalence of an abnormal CMR study between these groups (92 \% vs. $75 \%, p=0.27$ ).

miRNA findings in MD patients vs. controls

Plasma miRNA results are shown in Table 3. A significant up-regulation in MD patients compared to controls was found for seven out of 17 plasma miRNAs included in the analysis: miR-206 (91-fold increase, $p<0.0001$ ), miR-20b (1.5-fold increase, $p=0.048$ ), miR-222 (24-fold, $p<0.0001$ ), miR-26a (6-fold, $p<0.0001)$, miR-342 (33-fold, $p<0.0001$ ), miR-378a-3p (minimum 3000-fold; almost undetectable in controls, $p<0.0001$ ), miR-378a-5p (48-fold, $p<0.0001$ ). Additionally, a significant down-regulation was found for the three miRNAs: miR-221, miR-29a and miR-29c, all almost undetectable in the plasma of MD patients.

Among MD patients, none of the miRNAs differed significantly in patients with elevated vs. normal hs-Trop and NT-proBNP, respectively. Moreover, we did not detect any significant differences in miRNA expression in MD patients with mild myopathy (clinical degree $0-1$ as defined in our Methods section) compared to those with advanced/severe myopathy (clinical degree of 2-3) (Additional file 2: Table S2).

When we assessed the relationship between plasma miRNAs and age, significant but moderate correlations were found only for two of them: miR-26a (Spearman's rho $=+0.312, p=0.013$ ) and miR-378a-3p (Spearman's rho $=-0.299, p=0.017)$ in the MD group. In contrast, no significant relationship was found in controls regarding plasma miRNA levels and age.

\section{Assessment of CMR and miRNA findings considering all CMR results}

When MD patients with any abnormal CMR findings $(n=49 ; 78 \%)$ were compared to those without $(n=14$; $22 \%)$, there was no significant difference regarding age or MD type (Tables 4 and 5). An elevated hs-Trop plasma level was more frequently observed in patients with abnormal CMR findings compared to those without. However, only $57 \%(n=28)$ of those patients with pathological CMR findings also demonstrated elevation of hs-Trop. Moreover, there were no significant differences in NTproBNP levels between MD patients with abnormal CMR findings and those without.

As shown in Table 6, there were no significant differences in any of the measured plasma miRNAs in MD 
a

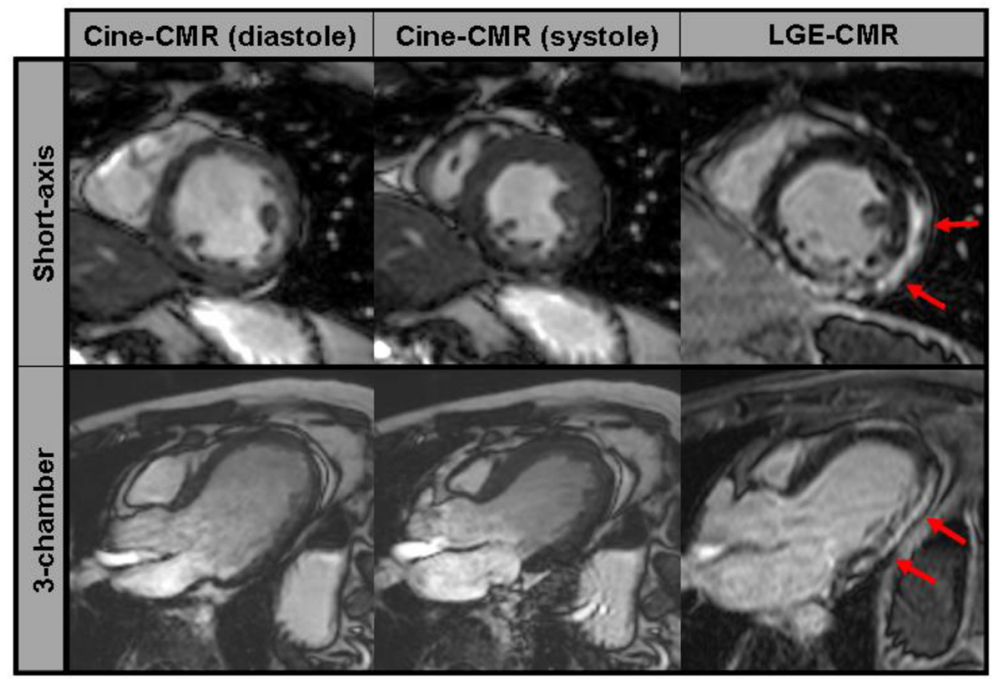

b

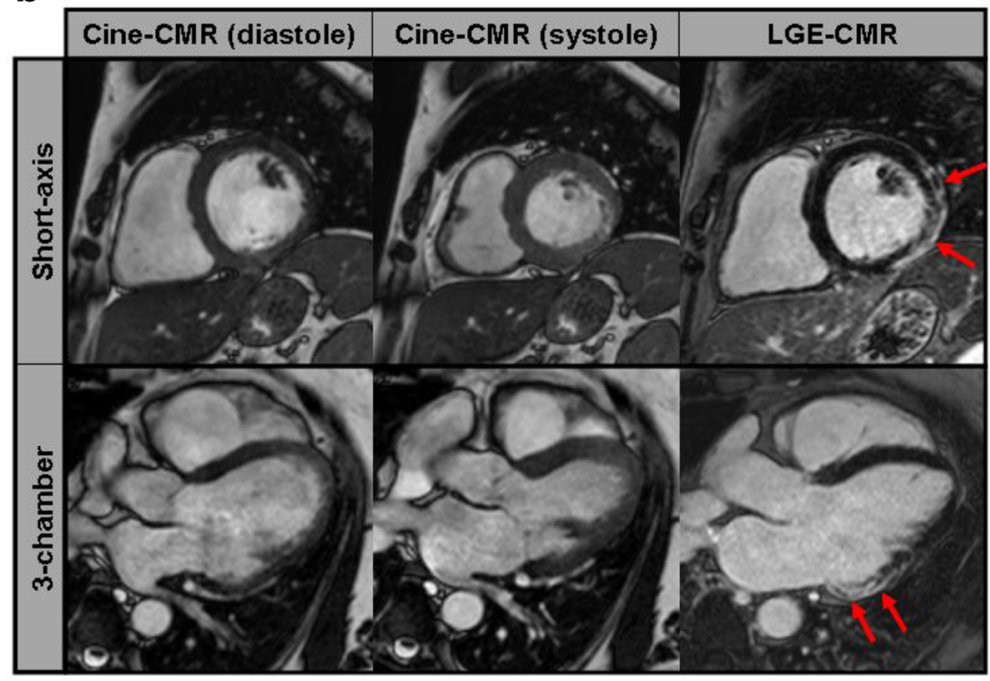

Fig. 1 Examples of cine- and late gadolinium enhancement (LGE)-CMR images of a patient with Duchenne (a) and Becker muscular dystrophy (b) in short-axis views (upper panels) and 3-chamber views (lower panels). In both, the same non-ischemic, predominantly subepicardial LGE pattern (arrows) indicating myocardial fibrosis can be seen.

patients with any abnormal CMR finding (comprising structural and/or functional impairments) compared to those with completely normal CMR studies.

\section{Assessment of CMR and miRNA findings according to "functional" CMR results}

Fifty-one percent $(n=32)$ of the MD patients showed a reduced LV and/or RV systolic function in the CMR study as measured by the respective EF. In this subgroup of MD patients with impaired systolic function, both LGE prevalence and extent were significantly increased compared to those MD patients with normal biventricular systolic function $(n=31 ; 49 \%)$ as depicted in Tables 7 and 8.
However, there were again no significant differences in any of the measured plasma miRNAs in MD patients with an impaired systolic function compared to those without (Table 9). The only significant correlation between a miRNA and LV-EF was a moderate negative one for miR-29c (Spearman's rho $=-0.323, p=0.010$ ).

\section{Assessment of CMR and miRNA findings according to "structural" CMR results}

In the next step, MD patients with presence of structural abnormalities - defined as presence of LGE $(n=48 ; 76 \%)$ were compared to those without any LGE ( $n=15 ; 24 \%)$. LGE-positive MD patients showed more frequently a reduced LV-EF (28 (58 \%) vs. 1 (7 \%); $p=0.001)$ and an 
Table 3 Plasma miRNA results in MD patients vs. controls

\begin{tabular}{lllc}
\hline $\begin{array}{l}\text { miRNA plasma } \\
\text { levels } \mathrm{s}^{\mathrm{a}}\left(/ 10^{3}\right)\end{array}$ & $N=63$ & Controls & $p$ value \\
\hline-206 & $17.20(4.29-63.96)$ & $0.19(0.12-0.64)$ & $<0.0001$ \\
$-144-5 p$ & $9.85(0.00-169.32)$ & $13.16(6.53-19.63)$ & 0.81 \\
$-146 b$ & $62.55(0.00-176.97)$ & $16.64(6.86-33.02)$ & 0.16 \\
$-15 b$ & $0.00(0.00-19.61)$ & $2.46(0.81-3.93)$ & 0.64 \\
-195 & $9.01(0.52-21.73)$ & $13.63(10.46-16.81)$ & 0.053 \\
$-20 b$ & $61.74(21.70-85.10)$ & $35.95(27.39-51.02)$ & $\mathbf{0 . 0 4 8}$ \\
-21 & $30.61(0.00-110.97)$ & $9.58(5.86-18.33)$ & 0.48 \\
-221 & $0.00(0.00-20.44)$ & $10.61(7.73-17.12)$ & $\mathbf{0 . 0 0 1}$ \\
-222 & $1923.17(881.56-5111.93)$ & $79.22(43.34-169.52)$ & $<0.0001$ \\
$-26 a$ & $293.34(121.00-554.69)$ & $49.40(26.58-63.10)$ & $<0.0001$ \\
$-29 a$ & $0.00(0.00-0.00)$ & $0.07(0.00-1.11)$ & $\mathbf{0 . 0 0 2}$ \\
$-29 c$ & $0.00(0.00-1.43)$ & $0.56(0.27-2.72)$ & $\mathbf{0 . 0 0 1}$ \\
-342 & $2427.02(1198.59-4185.82)$ & $73.89(45.30-171.19)$ & $<0.0001$ \\
$-378 a-3 p$ & $30(5.07-268.79)$ & $0.00(0.00-0.23)$ & $<0.0001$ \\
$-378 a-5 p$ & $27.57(8.84-81.04)$ & $0.58(0.30-1.79)$ & $<0.0001$ \\
-451 & $184.13(73.28-343.07)$ & $144.45(107.74-169.24)$ & 0.19 \\
-93 & $26.82(0.00-66.29)$ & $35.37(25.60-49.70)$ & 0.24 \\
\hline
\end{tabular}

$\left({ }^{a}\right)$ - Each miRNA plasma level was normalized to miR-16 plasma levels and thereafter multiplied by $10^{3}$ in order to increase readability

Bold text indicates a significant $p$-value of $<0.05$

elevated hs-Trop level (28 (58 \%) vs. 2 (13\%); $p=0.003$ ) compared to LGE-negative ones (Tables 10 and 11).

As illustrated in Table 12, a significant up-regulation of three miRNAs was observed in LGE-positive MD patients compared to LGE-negative ones: miR-222 (1.8fold, $p=0.035)$, miR-26a (2.1-fold, $p=0.03)$ and miR378a-5p (2.4-fold, $p=0.026$ ). There was no miRNA that was significantly down-regulated in the LGE-positive group compared to the LGE-negative one.
When the miRNA results of LGE-positive and LGEnegative patients were compared to controls, there were significant differences when all three groups were considered (Fig. 2a-c). However, when post hoc analysis for multiple group comparisons was performed, differences between MD patients with and without LGE were only borderline significant for any of the miRNAs - as expected in case of multiple testing in a small-sized study group. While in this post hoc comparison circulating miR-222 and miR-378a-5p levels were significantly higher both in LGE-positive and LGE-negative patients compared to controls $(p=0.003$ and $p<0.0001$ for miR222; $p=0.017$ and $p<0.0001$ for miR-378a-5p), a significant difference was noted only between LGE-positive patients and controls for miR-26a $(p<0.0001)$ - but not for LGE-negative ones vs. controls.

\section{Assessment of possible predictors of LGE presence}

In order to further characterize the association between myocardial scarring as detected by LGE-CMR and the three up-regulated plasma miRNAs, we first performed univariable logistic regression analyses for a series of potential predictors of LGE occurrence - including miR222, miR-26a and miR-378a-5p (Table 13). In this analysis, a significant association with LGE presence was found for the following parameters: a) CMR parameters such as LV-EF, LV-ESVi and LV-mass, b) the serum marker elevated hs-Trop and c) the miRNAs miR-222 and miR-26a.

In the next step, we performed a multivariable regression analysis focusing on four variables of most interest among those being statistically significant variables from the aforementioned univariable analysis (LV-EF, elevated hs-Trop, miR-222 and miR-26a). In this multivariable model, three independent predictors for LGE presence were found: a) LV-EF (OR 0.47, 95 \% CI 0.24-0.89, $p=$

Table 4 Patient characteristics and cardiac findings according to CMR results

\begin{tabular}{|c|c|c|c|c|}
\hline & MD & Normal CMR & Abnormal CMR & $p$ value \\
\hline & $N=63$ & $N=14$ & $N=49$ & \\
\hline Male, $n(\%)$ & $63(100)$ & $14(100)$ & $49(100)$ & 1.00 \\
\hline Age, years & $31 \pm 15$ & $25 \pm 18$ & $32 \pm 14$ & 0.11 \\
\hline $\mathrm{BMD}, n(\%)$ & $51(81)$ & $13(93)$ & $38(78)$ & 0.27 \\
\hline $\mathrm{BMI}, \mathrm{kg} / \mathrm{m}^{2}$ & $24 \pm 4$ & $23 \pm 6$ & $24 \pm 4$ & 0.75 \\
\hline Skeletal muscle status $0 / 1 / 2 / 3, n(\%)$ & $2(3) / 43(68) / 13(21) / 5(8)$ & $2(14) / 10(71) / 1(7) / 1(7)$ & $33(67) / 12(25) / 4(8) / 0(0)$ & 0.07 \\
\hline ACE inhibitor, $n(\%)$ & $16(25)$ & $1(7)$ & $15(31)$ & 0.09 \\
\hline Beta-blocker, n (\%) & $14(22)$ & $1(7)$ & $13(27)$ & 0.16 \\
\hline CK, U/L & $1226(653-2424)$ & $764(596-2411)$ & $1344(783-2463)$ & 0.24 \\
\hline Elevated hs-Trop, $n(\%)^{a}$ & $30(48)$ & $2(14)$ & $28(57)$ & 0.006 \\
\hline Elevated NT-proBNP, $n(\%)^{\mathrm{b}}$ & $3(5)$ & $0(0)$ & $3(6)$ & 1.00 \\
\hline
\end{tabular}

${ }^{\mathrm{a}}>14 \mathrm{pg} / \mathrm{mL} ;{ }^{\mathrm{b}}>450 \mathrm{pg} / \mathrm{mL}$

Bold text indicates a significant $p$-value of $<0.05$ 
Table 5 Cardiac findings according to CMR results

\begin{tabular}{lllll}
\hline & MD & Normal CMR & Abnormal CMR & $p$ value \\
& N=63 & $N=14$ & $N=49$ & \\
\hline LV-EDVi, ml/m² & $85 \pm 30$ & $72 \pm 19$ & $88 \pm 32$ & 0.08 \\
LV-ESVi, ml/m² & $41 \pm 21$ & $24 \pm 7$ & $46 \pm 21$ & $<\mathbf{0 . 0 0 0 1}$ \\
LV-mass, g/m² & $60 \pm 16$ & $52 \pm 11$ & $62 \pm 17$ & $\mathbf{0 . 0 3 7}$ \\
LV-EF, \% & $53 \pm 12$ & $67 \pm 6$ & $50 \pm 11$ & $<\mathbf{0 . 0 0 0 1}$ \\
RV-EF, \% & $53 \pm 9$ & $58 \pm 7$ & $52 \pm 9$ & $\mathbf{0 . 0 2 3}$ \\
LV-EF $<55 \%, n(\%)$ & $29(46)$ & $0(0)$ & $29(59)$ & NA \\
RV-EF $<45 \%, n(\%)$ & $10(16)$ & $0(0)$ & $10(20)$ & NA \\
LGE presence, $n(\%)$ & $48(76)$ & $0(0)$ & $48(98)$ & NA \\
LGE extent, \% & $7(1-17)$ & - & $10(4-24)$ & NA \\
\hline
\end{tabular}

Bold text indicates a significant $p$-value of $<0.05$

0.021), b) an elevated hs-Trop (OR 2559, 95 \% CI 2.97$22.04 \% 10^{5}, p=0.023$ ) and c) serum miR-222 levels (OR 938, $95 \%$ CI 3.56-24.73*10 $\left.{ }^{4}, p=0.016\right)$.

\section{Plasma miRNAs for the identification of MD patients with LGE presence}

As shown in Fig. 3a-c, the individual receiver operating characteristics curves (ROC) for the three significantly up-regulated miRNAs in MD patients with vs. without

Table 6 Plasma miRNA results in MD patients with normal vs. pathological CMR results

\begin{tabular}{llll}
\hline miRNA plasma & Normal CMR & Abnormal CMR & $p$ value \\
levels $\left(/ 10^{3}\right)$ & $N=14$ & $N=49$ & \\
\hline-206 & $14.36(3.07-49.46)$ & $21.76(4.82-72.71)$ & 0.68 \\
$-144-5 p$ & $8.94(0.00-163.41)$ & $9.85(0.00-171.21)$ & 0.97 \\
$-146 b$ & $84.15(40.30-169.65)$ & $38.75(0.00-177.31)$ & 0.25 \\
$-15 b$ & $7.44(0.00-21.87)$ & $0.00(0.00-18.78)$ & 0.43 \\
-195 & $9.76(1.53-19.84)$ & $8.31(0.57-21.25)$ & 0.86 \\
$-20 b$ & $49.38(29.88-79.03)$ & $65.38(19.43-86.37)$ & 0.66 \\
-21 & $71.91(2.89-107.05)$ & $11.48(0.00-113.69)$ & 0.61 \\
-221 & $0.00(0.00-35.26)$ & $0.00(0.00-11.27)$ & 0.87 \\
-222 & 1367.66 & 2230.30 & 0.09 \\
& $(689.45-1764.90)$ & $(964.52-5884.91)$ & \\
$-26 a$ & $173.94(47.45-324.42)$ & $326.81(126.07-602.29)$ & 0.63 \\
$-29 a$ & $0.00(0.00-0.00)$ & $0.00(0.00-0.00)$ & 0.94 \\
$-29 c$ & $0.00(0.00-2.24)$ & $0.00(0.00-0.56)$ & 0.93 \\
-342 & 1987.68 & 2639.34 & 0.16 \\
$-378 a-3 p$ & $(808.31-2723.93)$ & $(1333.85-5106.77)$ & \\
$-378 a-5 p$ & $14.15(0.00-273.74)$ & $39.74(5.89-290.67)$ & 0.39 \\
-451 & $17.32(2.66-40.41)$ & $39.29(13.24-101.0)$ & 0.06 \\
-93 & $24.46(0.38-55.52)$ & $26.95(0.00-69.92)$ & 0.93 \\
\hline
\end{tabular}

( ${ }^{\mathrm{a}}$ ) - Each miRNA plasma level was normalized to miR-16 plasma levels and thereafter multiplied by $10^{3}$ in order to increase readability

Bold text indicates a significant $p$-value of $<0.05$
LGE revealed areas under the curve (AUC) close to 0.70 . Sensitivities, specificities and overall accuracies for these miRNAs were as follows: a) miR-26a: 65,73 and $67 \%$ (cut-off value of 241.54*10-3), b) miR-222: 62, 80 and $65 \%$ (cut-off value of $1833.18 \% 10-3$ ) and c) miR-378a5p: 69, 60 and $67 \%$ (cut-off value of 18.84*10-3).

Furthermore, by combining these three miRNAs (miR26a, miR-222 and miR-378a-5p, Fig. 3d) as potential diagnostic signature for cardiomyopathy in MD patients, an improved AUC value of 0.74 was reached with a sensitivity of $70 \%$, a specificity of $73 \%$ and an overall accuracy of $70 \%$. In comparison, ROC analysis for the conventional serum marker hs-Trop regarding the identification of LGE-positive MD patients revealed an AUC value of 0.75 with a sensitivity of $63 \%$, a specificity of $87 \%$ and an overall accuracy of $65 \%$ (Fig. 3e). Moreover, there was no significant correlation between hs-Trop and each of the three miRNAs (miR-26a, miR-222 and miR-378a-5p).

\section{Discussion}

To the best of our knowledge, this is the first study that assessed the diagnostic value of circulating miRNAs for the detection and prediction of functional as well as structural cardiac impairments (that were assessed by comprehensive CMR studies) in MD patients. The present results indicate that some circulating miRNAs are differently expressed in the plasma of MD patients compared to healthy volunteers. However, for the first time we also identified three circulating miRNAs (miR26a, miR-222 and miR-378a-5p) that were significantly up-regulated in MD patients with proof of myocardial scarring (based on LGE-CMR) compared to those without - independent of the patients' age and skeletal muscle status. Interestingly, the association between the plasma profile of these three miRNAs and the presence of cardiac disease was only observed in case of structural changes (proof of myocardial scarring) - but not in case of exclusive functional abnormalities.

\section{Pathophysiology of cardiac involvement in MD}

As outlined previously, the detailed molecular pathomechanism leading to cardiac disease in MD patients is still to be elucidated, although the underlying genetic dystrophin defect can be identified easily by appropriate mutation screening [1]. Based on preclinical studies in dystrophin deficient animal models, early alterations in cardiomyocyte metabolism and signal transduction were suggested [20]. In addition, excessive intracellular calcium signalling and reactive oxygen species (ROS) generation with breakdown of the mitochondrial membrane potential were described and may constitute the link between the initial sarcolemmal injury due to dystrophin deficiency and mitochondrial dysfunctions. Of note, De Arcangelis et al. showed in an animal model that miRNAs are 
Table 7 Patient characteristics focusing on functional CMR results

\begin{tabular}{|c|c|c|c|c|}
\hline & \multirow{2}{*}{$\begin{array}{l}M D \\
N=63\end{array}$} & \multirow{2}{*}{$\begin{array}{l}\text { Normal LV and RV function } \\
N=31\end{array}$} & \multirow{2}{*}{$\begin{array}{l}\text { Impaired LV and/or RV function } \\
N=32\end{array}$} & \multirow[t]{2}{*}{$p$ value } \\
\hline & & & & \\
\hline Male, $n(\%)$ & $63(100)$ & $31(100)$ & $32(100)$ & 1.00 \\
\hline Age, years & $31 \pm 15$ & $27 \pm 15$ & $34 \pm 15$ & 0.11 \\
\hline BMD, $n(\%)$ & $51(81)$ & $26(84)$ & $25(78)$ & 0.75 \\
\hline $\mathrm{BMI}, \mathrm{kg} / \mathrm{m}^{2}$ & $24 \pm 4$ & $24 \pm 5$ & $24 \pm 3$ & 0.72 \\
\hline Skeletal muscle status $0 / 1 / 2 / 3, n(\%)$ & $2(3) / 43(68) / 13(21) / 5(8)$ & $2(7) / 23(74) / 5(16) / 1(3)$ & $20(63) / 8(25) / 4(13) / 0(0)$ & 0.20 \\
\hline ACE inhibitor, $n(\%)$ & $16(25)$ & $2(7)$ & $14(44)$ & 0.001 \\
\hline Beta-blocker, n (\%) & $14(22)$ & $4(13)$ & $10(31)$ & 0.13 \\
\hline $\mathrm{CK}, \mathrm{U} / \mathrm{L}$ & $1226(653-2424)$ & $1250(652-3043)$ & $1221(727-2291)$ & 0.86 \\
\hline Elevated hs-Trop, $n(\%)^{\mathrm{a}}$ & $30(48)$ & $8(26)$ & $22(67)$ & 0.001 \\
\hline Elevated NT-proBNP, $n(\%)^{\mathrm{b}}$ & $3(5)$ & $0(0)$ & $3(9)$ & 0.24 \\
\hline
\end{tabular}

a $>14 \mathrm{pg} / \mathrm{mL}^{\circ}{ }^{\text {b }}>450 \mathrm{pg} / \mathrm{mL}$

Bold text indicates a significant $p$-value of $<0.05$

involved in the regulation of the dystrophin-glycoprotein complex components in dystrophic muscles of the $\mathrm{mdx}$ mice. Especially, miR-222 expression was increased in dystrophic muscle and led to a decrease of the b1-syntrophin expression [21]. Taken together, the fragility of the cell membrane that is caused by deficient sarcolemmal dystrophin may predispose not only skeletal muscle cells but also cardiomyocytes to metabolic dysfunctions, which in turn may be enhanced by mechanical stress.

In recent years, CMR imaging has gained wide acceptance for non-invasive evaluation of ischaemic as well as non-ischaemic cardiomyopathies since multi-parametric CMR does not only allow the assessment of functional parameters (such as systolic function) but also enables a non-invasive assessment of myocardial scarring based on

Table 8 Cardiac findings focusing on functional CMR results

\begin{tabular}{|c|c|c|c|c|}
\hline & $\mathrm{MD}$ & $\begin{array}{l}\text { Normal LV and } \\
\text { RV function }\end{array}$ & $\begin{array}{l}\text { Impaired LV and/or } \\
\text { RV function }\end{array}$ & $p$ value \\
\hline & $N=63$ & $N=31$ & $N=32$ & \\
\hline LV-EDVi, $\mathrm{ml} / \mathrm{m}^{2}$ & $85 \pm 30$ & $73 \pm 18$ & $96 \pm 35$ & 0.003 \\
\hline LV-ESVi, $\mathrm{ml} / \mathrm{m}^{2}$ & $41 \pm 21$ & $27 \pm 9$ & $54 \pm 21$ & $<0.0001$ \\
\hline LV-mass, $\mathrm{g} / \mathrm{m}^{2}$ & $60 \pm 16$ & $53 \pm 11$ & $66 \pm 18$ & 0.001 \\
\hline LV-EF, \% & $53 \pm 12$ & $63 \pm 6$ & $44 \pm 9$ & NA \\
\hline RV-EF, \% & $53 \pm 9$ & $58 \pm 7$ & $49 \pm 9$ & NA \\
\hline $\begin{array}{l}\text { LV-EF }<55 \%, \\
n(\%)\end{array}$ & $29(46)$ & $0(0)$ & $29(91)$ & NA \\
\hline $\begin{array}{l}\text { RV-EF }<45 \%, \\
n(\%)\end{array}$ & $10(16)$ & $0(0)$ & $10(31)$ & NA \\
\hline $\begin{array}{l}\text { LGE presence, } \\
n(\%)\end{array}$ & $48(76)$ & $17(55)$ & $31(97)$ & $<0.0001$ \\
\hline LGE extent, \% & $7(1-17)$ & $1(0-6)$ & $16(8-32)$ & $<0.0001$ \\
\hline $\begin{array}{l}\text { Abnormal CMR, } \\
n(\%)\end{array}$ & 49 (78) & $17(55)$ & $32(100)$ & NA \\
\hline
\end{tabular}

Bold text indicates a significant $p$-value of $<0.05$
LGE-imaging or T1-mapping [22]. Within the last years, the distribution pattern of myocardial scarring in MD patients (as well as female MD carriers) was assessed based on such comprehensive CMR studies and it could be consistently shown that the posterolateral LV wall segments represent the first and most extensive sites of myocardial

Table 9 Plasma miRNA results in MD patients with normal vs. impaired systolic function

\begin{tabular}{llll}
\hline $\begin{array}{l}\text { miRNA plasma } \\
\text { levels }{ }^{\mathrm{a}}\left(/ 10^{3}\right)\end{array}$ & $\begin{array}{l}\text { Normal LV and } \\
\text { RV function }\end{array}$ & $\begin{array}{l}\text { Impaired LV and/or } \\
\text { RV function }\end{array}$ & $p$ value \\
& $N=31$ & $N=32$ & \\
\hline-206 & $31.77(4.83-79.62)$ & $11.27(3.52-50.27)$ & 0.58 \\
$-144-5 p$ & $33.14(0.00-525.08)$ & $0.00(0.00-98.72)$ & 0.29 \\
$-146 b$ & $74.79(0.00-173.85)$ & $29.92(0.00-177.79)$ & 0.60 \\
$-15 b$ & $0.00(0.00-18.15)$ & $0.18(0.00-21.81)$ & 0.77 \\
-195 & $9.01(0.35-18.62)$ & $8.93(1.90-22.0)$ & 0.66 \\
$-20 b$ & $53.99(25.84-83.60)$ & $65.45(19.35-87.12)$ & 0.66 \\
-21 & $42.09(0.00-102.71)$ & $13.65(0.00-150.01)$ & 0.41 \\
-221 & $0.00(0.00-18.27)$ & $0.00(0.00-18.55)$ & 0.36 \\
-222 & 1801.09 & 2006.29 & 0.79 \\
& $(1215.81-3491.60)$ & $(638.43-6059.36)$ & \\
$-26 a$ & $283.14(110.62-512.14)$ & $362.31(121.25-622.72)$ & 0.36 \\
$-29 a$ & $0.00(0.00-0.00)$ & $0.00(0.00-0.05)$ & 0.39 \\
$-29 c$ & $0.00(0.00-0.47)$ & $0.00(0.00-2.16)$ & 0.27 \\
-342 & 2249.28 & 2570.15 & 0.54 \\
$-378 a-3 p$ & $(1294.10-3353.27)$ & $(888.62-5565.11)$ & \\
$-378 a-5 p$ & $28.84(6.04-155.98)$ & $32.92(1.76-301.61)$ & 0.98 \\
-451 & $27.57(12.50-56.49)$ & $26.02(7.00-84.56)$ & 0.78 \\
-93 & $184.13(100.07-349.44)$ & $171.93(22.68-307.38)$ & 0.77 \\
\hline
\end{tabular}

$\left({ }^{\mathrm{a}}\right)$ - Each miRNA plasma level was normalized to miR-16 plasma levels and thereafter multiplied by $10^{3}$ in order to increase readability Bold text indicates a significant $p$-value of $<0.05$ 
Table 10 Patient characteristics focusing on structural CMR results

\begin{tabular}{|c|c|c|c|c|}
\hline & MD & LGE-negative & LGE-positive & $p$ value \\
\hline & $N=63$ & $N=15$ & $N=48$ & \\
\hline Male, $n(\%)$ & $63(100)$ & $15(100)$ & $48(100)$ & 1.00 \\
\hline Age, years & $31 \pm 15$ & $25 \pm 17$ & $32 \pm 14$ & 0.13 \\
\hline $\mathrm{BMD}, n(\%)$ & $51(81)$ & $14(93)$ & $37(77)$ & 0.26 \\
\hline $\mathrm{BMI}, \mathrm{kg} / \mathrm{m}^{2}$ & $24 \pm 4$ & $23 \pm 5$ & $24 \pm 4$ & 0.38 \\
\hline Skeletal muscle status $0 / 1 / 2 / 3, n(\%)$ & $2(3) / 43(68) / 13(21) / 5(8)$ & $2(13) / 11(73) / 1(7) / 1(7)$ & $32(67) / 12(25) / 4(8) / 0(0)$ & 0.06 \\
\hline ACE inhibitor, $n(\%)$ & $16(25)$ & $1(7)$ & $15(31)$ & 0.09 \\
\hline Beta-blocker, $n(\%)$ & $14(22)$ & $1(7)$ & $13(27)$ & 0.16 \\
\hline CK, U/L & $1226(653-2424)$ & $773(607-2187)$ & $1358(797-2481)$ & 0.18 \\
\hline Elevated hs-trop, $n(\%)^{a}$ & $30(48)$ & $2(13)$ & $28(58)$ & 0.003 \\
\hline Elevated NT-proBNP, $n(\%)^{\mathrm{b}}$ & $3(5)$ & $0(0)$ & $3(6)$ & 1.00 \\
\hline
\end{tabular}

${ }^{\mathrm{a}}>14 \mathrm{pg} / \mathrm{mL} ;{ }^{\mathrm{b}}>450 \mathrm{pg} / \mathrm{mL}$

Bold text indicates a significant $p$-value of $<0.05$

fibrosis in MD patients - as well as their female MD carrier relatives [17, 23-25] (Fig. 1). Moreover, we could recently show that a "transmural" pattern of myocardial fibrosis independently predicts the occurrence of adverse cardiac events in DMD/BMD patients [23].

\section{Conventional serum markers of cardiac disease in MD patients}

Today, measurement of serum BNP levels is routinely performed in patients presenting with heart failure symptoms and/or suspected cardiomyopathy (e.g. dilated cardiomyopathy) since normal BNP values allow to elegantly rule out congestive heart failure whereas elevated BNP values (in patients with normal renal function) point to an underlying cardiac disease and necessitate further cardiac examinations such as echocardiography and/or CMR [9]. Interestingly, in the present study (comprising $63 \mathrm{MD}$ patients), elevated Nt-proBNP levels were measured in three (5\%) MD patients only - although any abnormal cardiac findings were detected in $49(78 \%)$ and a reduced LV-EF

Table 11 Cardiac findings focusing on structural CMR results

\begin{tabular}{lllll}
\hline & MD & LGE-negative & LGE-positive & $p$ value \\
& $N=63$ & $N=15$ & $N=48$ & \\
\hline LV-EDVi, ml/m² & $85 \pm 30$ & $72 \pm 18$ & $89 \pm 32$ & 0.06 \\
LV-ESVi, ml/m² & $41 \pm 21$ & $25 \pm 7$ & $46 \pm 22$ & $<\mathbf{0 . 0 0 0 1}$ \\
LV-mass, g/m² & $60 \pm 16$ & $51 \pm 11$ & $62 \pm 17$ & $\mathbf{0 . 0 1 8}$ \\
LV-EF, \% & $53 \pm 12$ & $66 \pm 6$ & $50 \pm 11$ & $<\mathbf{0 . 0 0 0 1}$ \\
RV-EF, \% & $53 \pm 9$ & $56 \pm 10$ & $52 \pm 9$ & 0.15 \\
LV-EF <55 \%, n (\%) & $29(46)$ & $1(7)$ & $28(58)$ & $\mathbf{0 . 0 0 1}$ \\
RV-EF <45 \%, n (\%) & $10(16)$ & $1(7)$ & $9(19)$ & 0.43 \\
LGE extent, \% & $7(1-17)$ & - & $10(5-24)$ & NA \\
Abnormal CMR, n (\%) & $49(78)$ & $1(7)$ & $48(100)$ & NA \\
\hline
\end{tabular}

Bold text indicates a significant $p$-value of $<0.05$ was observed in 29 (46\%) of them by CMR. Hence, measurement of serum BNP is not very sensitive for diagnosing or ruling out cardiac disease in MD patients. As discussed previously, one explanation for this observation may be that serum BNP levels are supposed to reflect rapid changes of pressure gradients in the atria and ventricles and one may argue that the underlying pathophysiology in

Table 12 Plasma miRNA results in MD patients with vs. without presence of LGE

\begin{tabular}{|c|c|c|c|}
\hline \multirow{2}{*}{$\begin{array}{l}\text { miRNA plasma } \\
\text { levels }^{a}\left(/ 10^{3}\right)\end{array}$} & \multirow{2}{*}{$\begin{array}{l}\text { LGE-negative } \\
N=15\end{array}$} & \multirow{2}{*}{$\begin{array}{l}\text { LGE-positive } \\
N=48\end{array}$} & \multirow[t]{2}{*}{$p$ value } \\
\hline & & & \\
\hline-206 & $13.18(3.69-43.72)$ & $22.85(4.56-74.83)$ & 0.54 \\
\hline$-144-5 p$ & $15.70(0.00-159.39)$ & $4.93(0.00-191.57)$ & 0.95 \\
\hline$-146 b$ & $81.86(32.74-162.66)$ & $44.23(0.00-177.79)$ & 0.32 \\
\hline$-15 b$ & $7.42(0.00-21.10)$ & $0.00(0.00-19.01)$ & 0.41 \\
\hline-195 & $10.50(2.70-17.48)$ & $8.19(0.54-21.93)$ & 0.75 \\
\hline$-20 b$ & 46.56 (31.69-74.64) & 65.45 (19.35-86.58) & 0.58 \\
\hline-21 & $67.48(5.74-106.23)$ & $11.82(0.00-115.78)$ & 0.65 \\
\hline-221 & $0.00(0.00-32.92)$ & $0.00(0.00-10.03)$ & 0.66 \\
\hline-222 & $\begin{array}{l}1288.92 \\
(355.69-1728.72)\end{array}$ & $\begin{array}{l}2303.59 \\
(1102.54-6059.36)\end{array}$ & 0.035 \\
\hline$-26 a$ & 165.39 (36.75-291.31) & 345.01 (139.78-616.20) & 0.030 \\
\hline$-29 a$ & $0.00(0.00-.09)$ & $0.00(0.00-0.00)$ & 0.61 \\
\hline$-29 c$ & $0.00(0.00-2.14)$ & $0.00(0.00-.65)$ & 0.89 \\
\hline-342 & $\begin{array}{l}1906.24 \\
(491.08-2603.63)\end{array}$ & $\begin{array}{l}2668.49 \\
(1512.77-5205.06)\end{array}$ & 0.07 \\
\hline$-378 a-3 p$ & $13.18(0.00-155.98)$ & $40.31(8.05-301.61)$ & 0.26 \\
\hline$-378 a-5 p$ & $16.99(1.04-36.13)$ & $41.23(14.62-102.03)$ & 0.026 \\
\hline-451 & $106.88(64.56-273.40)$ & 207.05 (100.16-340.80) & 0.29 \\
\hline-93 & $23.85(0.77-52.24)$ & $27.17(0.00-70.37)$ & 0.89 \\
\hline
\end{tabular}

$\left.{ }^{\mathrm{a}}\right)$ - Each miRNA plasma level was normalized to miR-16 plasma levels and thereafter multiplied by $10^{3}$ in order to increase readability Bold text indicates a significant $p$-value of $<0.05$ 


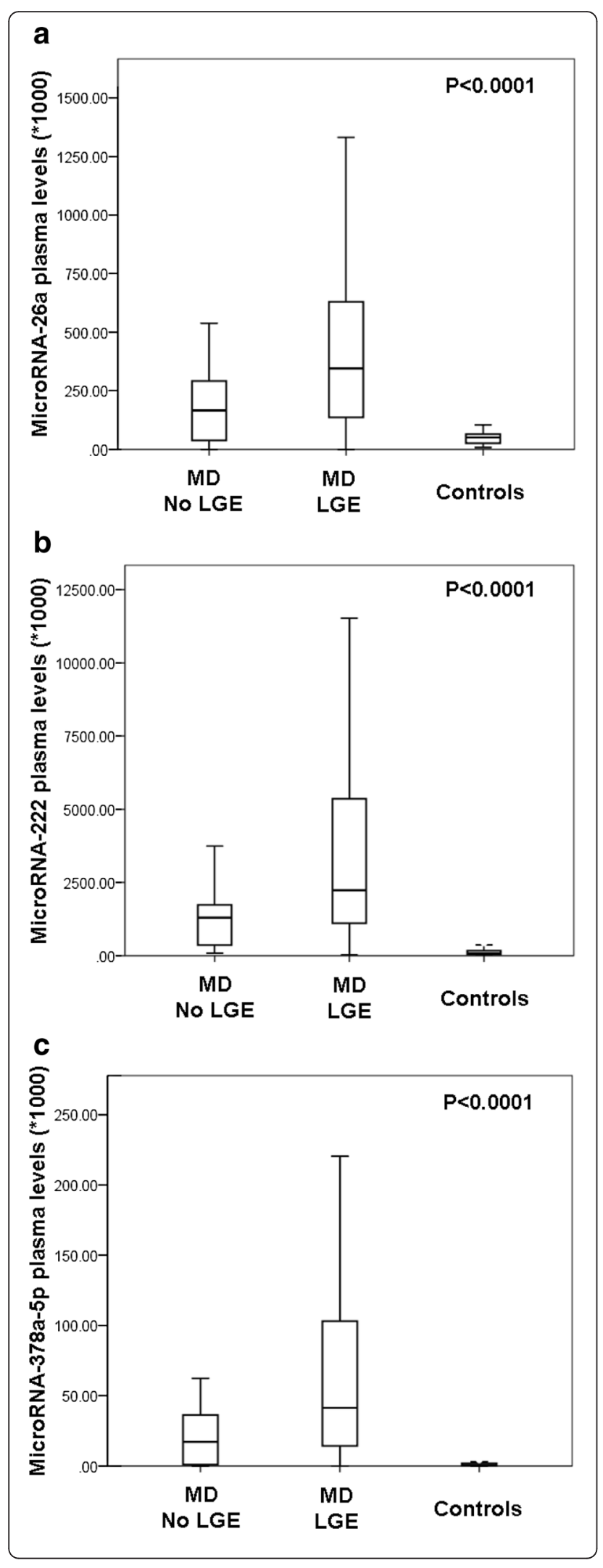

Fig. 2 Expression of circulating miRNA-26a (a), miRNA-222 (b) and miR-378a-5p (c) in plasma of LGEnegative ( $N=15)$ and LGE-positive $(N=48)$ muscular dystrophy patients as well as controls $(N=26)$. Each miRNA plasma level was normalized to miR-16 plasma levels and thereafter multiplied by 103 in order to increase readability. LGE: late gadolinium enhancement

MD patients is mainly driven by continuous myocardial cell death (due to dystrophin absence or fragility) resulting secondarily in a continuous and adapted ventricular enlargement with progressive decrease of systolic function, however, without quick changes in atrial/ventricular filling pressures $[10,26,27]$.

Furthermore, measurement of serum troponin is routinely performed nowadays not only in patients with acute chest pain syndromes but also in those with (suspected) non-ischemic cardiomyopathy for further detailed classification and risk stratification of such patients $[28,29]$. In particular, an elevated level of high-sensitive troponin is believed to be an accurate serum marker of myocardial cell death in various acute settings. In the present study, an elevated serum level of hs-Trop was detected in 30 (48 \%) MD patients only - although (as aforementioned) any abnormal cardiac findings were detected in 49 (78 \%) and proof of myocardial scarring/damage in $48(76 \%)$ of the study MD patients by CMR. Hence, measurement of serum hs-Trop in MD patients does not allow to detect all of those MD patients who have at least some structural cardiac abnormalities - as can be depicted by LGE-CMR. Moreover, even hs-Trop is not $100 \%$ specific

Table 13 Univariable analysis regarding predictors for LGE presence

\begin{tabular}{|c|c|c|}
\hline Variable $(N=63)$ & OR $(95 \% \mathrm{Cl})$ & $p$ value \\
\hline Age, years & $1.03(0.99-1.08)$ & 0.13 \\
\hline BMD, $n(\%)$ & $4.16(0.49-35.28)$ & 0.19 \\
\hline Skeletal muscle status 0/1/2/3, n (\%) & $2.83(0.84-9.50)$ & 0.09 \\
\hline $\log _{10} \mathrm{CK}, \mathrm{U} / \mathrm{L}$ & $1.69(0.39-7.36)$ & 0.48 \\
\hline LV-EDVi, $\mathrm{ml} / \mathrm{m}^{2}$ & $1.02(0.99-1.05)$ & 0.06 \\
\hline LV-ESVi, $\mathrm{ml} / \mathrm{m}^{2}$ & $1.11(1.04-1.19)$ & 0.003 \\
\hline LV-mass, $\mathrm{g} / \mathrm{m}^{2}$ & $1.06(1.01-1.11)$ & 0.024 \\
\hline LV-EF, \% & $0.81(0.71-0.91)$ & 0.001 \\
\hline Elevated hs-Trop, $n(\%)^{\mathrm{a}}$ & $9.1(1.85-44.87)$ & 0.007 \\
\hline $\operatorname{miR}-222\left(/ 10^{3}\right)^{\mathrm{c}, \mathrm{d}}$ & $2.51(1.01-6.21)$ & 0.047 \\
\hline $\operatorname{miR}-378 a-5 p\left(/ 10^{3}\right)^{c, d}$ & $1.00(0.99-1.01)$ & 0.86 \\
\hline $\operatorname{miR}-26 a\left(/ 10^{3}\right)^{c}$ & $12.86(1.07-154.34)$ & 0.044 \\
\hline
\end{tabular}

${ }^{\mathrm{a}}>14 \mathrm{pg} / \mathrm{mL}$

$\left({ }^{b}\right)$ - Each miRNA plasma level was normalized to miR-16 plasma levels and thereafter multiplied by $10^{3}$ in order to increase readability

( $\left.{ }^{c}\right)-\log _{10}$ transformed before introduced in the regression analysis Bold text indicates a significant $p$-value of $<0.05$ 

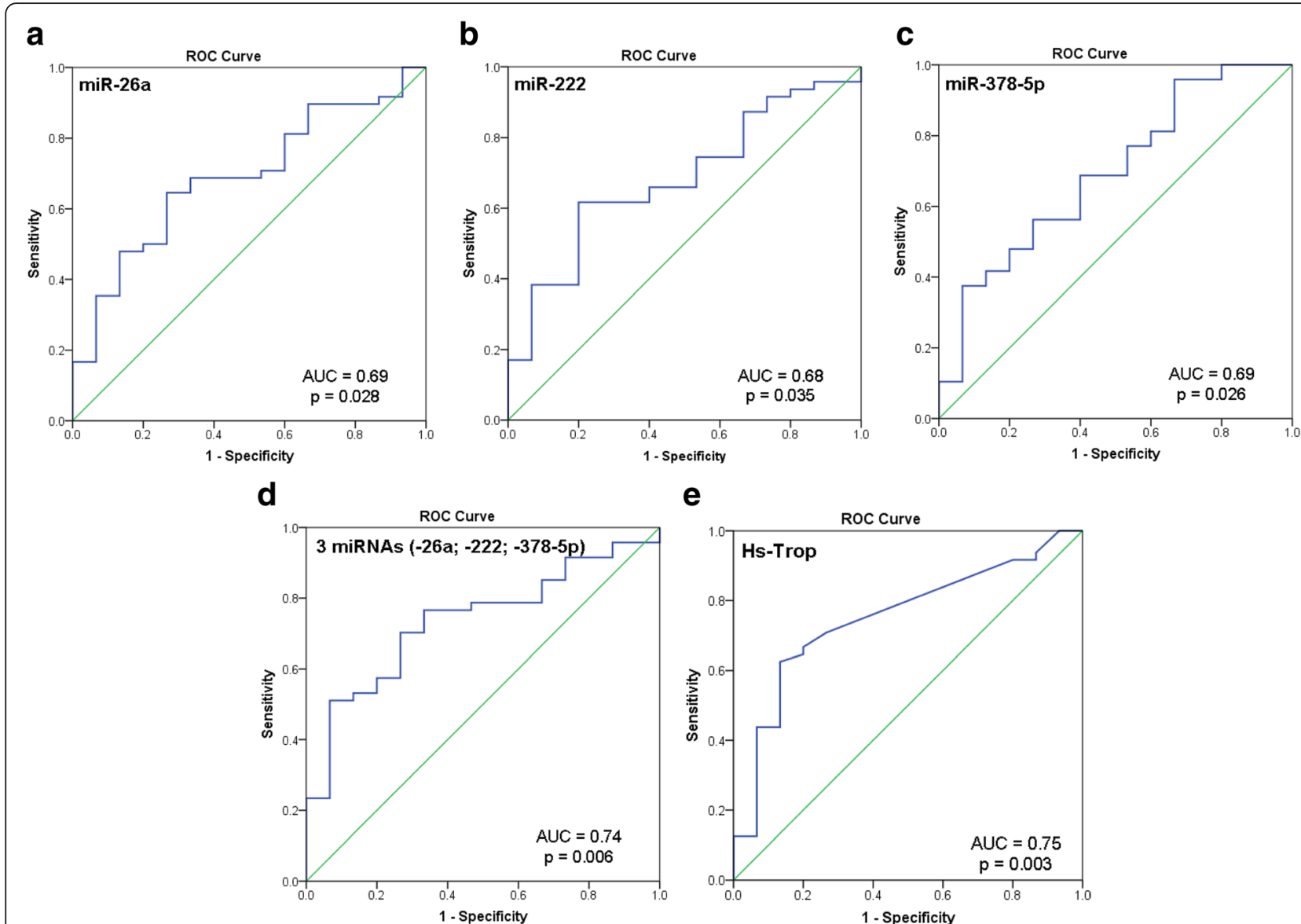

Fig. 3 Receiver operating characteristic (ROC) analysis of individual (a-c) and combined (d) plasma miRNA-26a, miRNA-222 and miR-378a-5p to discriminate LGE-negative $(N=15)$ from LGE-positive $(N=48)$ muscular dystrophy patients. Each miRNA plasma level was normalized to miR-16 plasma levels and thereafter multiplied by $10^{3}$ in order to increase readability. e ROC analysis for the conventional serum marker high-sensitive troponin (hs-Trop). LGE, late gadolinium enhancement. AUC, area under the curve

for cardiac disease and may also originate from cell death of skeletal muscle myocytes - particularly in patients with neuromuscular disorders and advanced skeletal myopathy. Taken together, a normal hs-Trop level in a MD patient does not always rule out cardiac disease and in contrast, an elevated hs-Trop level does not necessarily indicate the presence of cardiac abnormalities. Therefore, novel (more sensitive as well as more specific) markers are wanted for early and accurate diagnosis of cardiac involvement in MD patients.

\section{Circulating miRNAs as novel biomarkers of cardiac disease in MD patients}

Recent advances in molecular diagnostics have shown that circulating miRNAs can be used as diagnostic as well as prognostic biomarkers for different cardiovascular diseases $[14,15]$. Therefore, we tested the hypothesis that the pattern of plasma miRNA expression would also allow the diagnosis and prediction of cardiac disease in MD patients. In a first step, we searched for those miRNAs that were known to be associated with either cardiovascular diseases and/or DMD/BMD based on the available literature data [30-38]. Compared to our control group, we identified seven significantly up-regulated plasma miRNAs (miR-206, miR-20b, miR-222, miR-26a, miR-342, miR378a-3p, miR-378a-5p) and three down-regulated miRNAs (miR-221, miR-29a and miR-29c) in MD patients (Table 3). Since we did not detect any significant differences in miRNA expression in MD patients with mild myopathy (clinical degree $0-1$ as defined in our Methods section) compared to those with advanced/severe myopathy (clinical degree of 2-3), the aforementioned seven up-regulated and three down-regulated miRNAs do not seem to reflect the skeletal myopathy status of our MD patients.

In the next step, we looked at those MD patients who had any abnormal CMR findings compared to those with completely normal CMR results. Interestingly, there were no significant differences in any of the measured plasma miRNAs (Table 6). Then, we focused first on "functional" 
cardiac parameters and thereafter on "structural" ones. Again, we did not detect significant differences in any of the measured plasma miRNAs in MD patients when those with an impaired systolic function were compared to those without (Table 9). However, patients with proof of myocardial scarring (LGE-positive) compared to those without (LGE-negative) showed a significant up-regulation of three miRNAs (miR-222, miR-26a and miR-378a-5p, Table 12). Importantly, there was no significant difference in the degree of skeletal myopathy or serum CK in LGEpositive MD patients compared to LGE-negative ones. Hence, the elevation of these three miRNAs in LGEpositive MD patients was not (only) caused by skeletal myopathy, but rather reflected cardiac disease.

Additional univariable analyses revealed a significant and substantial association between the expression level of two miRNAs (miR-222 and miR-26a) and the presence of myocardial scarring. Furthermore, multivariable regression analysis revealed three independent predictors for the presence of LGE - one being a miRNA (miR-222) in addition to a clinical (LV-EF) and a laboratory marker (elevated hsTrop). Taken together, an up-regulation of three circulating miRNAs miR-222, miR-26a and miR-378a-5p is a useful signal for the presence of cardiac (structural) disease in MD patients - with miR-222 being the strongest and most important biomarker.

\section{Diagnostic value of plasma miRNAs compared to hs-Trop} In the present study, serum hs-Trop was a significant predictor of myocardial scarring - similar to miR-222. For further evaluation of the diagnostic value of the detected miRNAs in comparison to hs-Trop, we performed ROC analyses regarding the identification of LGE-positive MD patients. Area under the curve (AUC) values for the individual miRNAs (miR-222, miR-26a and miR-378a-5p) were close to 0.70 . However, combining these three miRNAs resulted in an improved AUC value of 0.74 - compared to 0.75 in case of hs-Trop. However, the respective sensitivity, specificity and overall accuracy was 70,73 and $70 \%$ for the combination of these three miRNAs compared to 63,87 and $65 \%$ in case of hs-Trop. Hence, a combined approach based on the measurement of the three plasma miRNAs miR-222, miR-26a and miR-378a$5 p$ seems to be at least as sensitive and accurate for the non-invasive diagnosis of cardiac disease in MD patients as the conventional hs-Trop measurement. Moreover, hsTrop elevations may also have extra-cardiac causes (e.g. renal failure or myositis) - which so far were not described for the three identified circulating miRNAs. In future studies, the potential superior diagnostic as well as prognostic value of a miRNA-based approach for diagnosis of cardiomyopathy compared to established measurements (e.g. hs-Trop) needs to be evaluated in larger study groups.

\section{Comparison of present miRNA results to previous literature data}

In the last years, several studies have been published addressing both pre-clinical animal models and humans as well as different biological materials such as skeletal muscle, heart muscle and serum/plasma. Previously published studies that (amongst others) addressed the three miRNAs miR-222, miR-26a and miR-378a-5p are summarized in the Additional file 3: Table S3. For example, miRNA analyses in the skeletal muscle of mdx mice and DMD patients revealed an up-regulation of miRNAs associated with muscle regeneration (e.g. miR-206) and inflammation (e.g. miR222 ) and a down-regulation of miRNAs associated with muscle degeneration (e.g. miR-29c) [31].

Recently, Jeanson-Leh et al. analysed circulating miRNA profiles in golden retriever muscular dystrophy dogs and also evaluated the association of miRNA to cardiac disease [33]. Importantly, evaluation of cardiac disease was based only on functional analyses using echocardiography. Although a dysregulation of numerous miRNAs (such as miR-1, miR-95, miR-133, miR-208a/b, miR-206, miR-378 and miR-499) was detected in the serum of these dogs, there was no correlation between any miRNAs and cardiac functional parameters in this model. In agreement with this study, we also detected a dysregulation of some circulating miRNAs (e.g. miR-206 and miR-378) in the plasma of MD patients. Notably, we elucidated only a significant association between miRNA and cardiac involvement in MD patients when we looked specifically at structural - but not functional - abnormalities using myocardial scarring based on LGE-CMR. Obviously, miRNA profiling may improve the understanding of the pathophysiology of a certain disease if in depth phenotypic characterisation is considered.

\section{Limitations}

Since the number of study patients was limited, replication of our data in an independent cohort is mandatory to demonstrate clinical utility. Moreover, in the present study we focused on selected miRNAs with evidence from previous studies. Thus, it cannot be excluded that additional miRNAs might even improve the predictive ability of the identified miRNA signature e.g. by comprehensive microarray profiling for miRNA expression. Finally, this was a pilot study and the results need verification in a larger cohort, with a multi-center approach and with consideration of clinical outcomes to define the respective role of miRNAs.

\section{Conclusions}

Up-regulation of circulating miRNAs miR-222, miR-26a and miR-378a-5p indicates the presence of myocardial scars in MD patients. Plasma miR-222 appears to be a promising novel biomarker reflecting structural - but not functional - cardiac alterations in MD patients. 


\section{Additional files}

Additional file 1: Table S1. Primer information. (DOC $67 \mathrm{~kb}$ )

Additional file 2: Table S2. Plasma miRNA results in MD patients according to the severity of skeletal myopathy. (DOC $40 \mathrm{~kb}$ )

Additional file 3: Table S3. Summary of published data regarding miR-26a, miR-222 and miR-378-a5p. (DOC $126 \mathrm{~kb}$ )

\section{Abbreviations}

BMD: Becker muscular dystrophy; CK: creatine kinase; CMR: cardiovascular magnetic resonance; DMD: Duchenne muscular dystrophy; hs-Trop: highsensitive troponin; LGE: late-gadolinium-enhancement; LV: left ventricle; LVEDV: left ventricular end-diastolic volume; LV-EF: left ventricular ejection fraction; LV-ESV: left ventricular end-systolic volume; MD: muscular dystrophy; NT-proBNP: brain natriuretic peptide; RV: right ventricle; RV-EDV: right ventricular end-diastolic volume; SSFP: steady-state-free-precession.

\section{Competing interests}

The authors declare that they have no competing interests.

\section{Authors' contributions}

SB carried out the molecular genetic studies and performed the statistical analysis; AF performed the CMR studies, performed the statistical analysis and helped to draft the manuscript; AP performed the CMR studies; SR participated in performing the CMR studies and collected the blood specimens; JW helped to draft the manuscript; US participated in the design of the study and helped to draft the manuscript; MS participated in the design of the study and helped to draft the manuscript; ES participated in the design and coordination of the study and helped to draft the manuscript; AY conceived the study, and participated in its design and coordination and helped to draft the manuscript. All authors read and approved the final manuscript.

\section{Acknowledgements}

We would like to acknowledge the expert technical assistance of Ursula Waldherr and Andrea Jarmuth.

\section{Funding sources}

This work was supported by the Robert-Bosch-Foundation (Stuttgart, Germany) and the Deutsche Forschungsgemeinschaft (Bonn, Germany) grant DFG $\mathrm{SCH} 858 / 1-2$

\section{Author details}

${ }^{1}$ Dr. Margarete Fischer-Bosch Institute of Clinical Pharmacology, Stuttgart, Germany. ${ }^{2}$ Department of Cardiovascular Medicine, University Hospital Münster, Albert-Schweitzer-Campus 1, building A1, 48149 Münster, Germany. ${ }^{3}$ Division of Cardiology, Robert-Bosch-Hospital, Stuttgart, Germany. ${ }^{4}$ Department of Clinical Pharmacology, University Hospital Tübingen, Tübingen, Germany. ${ }^{5}$ Department of Biochemistry and Pharmacy, University Tübingen, Tübingen, Germany.

Received: 19 November 2015 Accepted: 21 April 2016 Published online: 06 May 2016

\section{References}

1. Yilmaz A, Sechtem U. Cardiac involvement in muscular dystrophy: advances in diagnosis and therapy. Heart. 2012;98(5):420-9.

2. Verhaert D, Richards K, Rafael-Fortney JA, Raman SV. Cardiac involvement in patients with muscular dystrophies: magnetic resonance imaging phenotype and genotypic considerations. Circ Cardiovasc Imaging. 2011;4(1):67-76.

3. Finsterer J, Stollberger C. The heart in muscular dystrophies. Z Kardiol. 2005:94(11):791-2.

4. Hermans MC, Pinto YM, Merkies IS, de Die-Smulders CE, Crijns HJ Faber CG. Hereditary muscular dystrophies and the heart. Neuromuscul Disord. 2010;20(8):479-92.

5. Mavrogeni S, Papavasiliou A, Skouteli E, Magoutas A, Dangas G. Cardiovascular magnetic resonance imaging evaluation of two families with Becker muscular dystrophy. Neuromuscul Disord. 2010;20(11):717-9.

6. Florian A, Ludwig A, Rosch S, Yildiz H, Sechtem U, Yilmaz A. Myocardial fibrosis imaging based on T1-mapping and extracellular volume fraction
(ECV) measurement in muscular dystrophy patients: diagnostic value compared with conventional late gadolinium enhancement (LGE) imaging Eur Heart J Cardiovasc Imaging. 2014;15(9):1004-12.

7. Ikeda S, Kong SW, Lu J, et al. Altered microRNA expression in human heart disease. Physiol Genomics. 2007;31(3):367-73.

8. Matkovich SJ, Van Booven DJ, Youker KA, et al. Reciprocal regulation of myocardial microRNAs and messenger RNA in human cardiomyopathy and reversal of the microRNA signature by biomechanical support. Circulation. 2009;119(9):1263-71.

9. McMurray JJ, Adamopoulos S, Anker SD, et al. ESC guidelines for the diagnosis and treatment of acute and chronic heart failure 2012: The Task Force for the Diagnosis and Treatment of Acute and Chronic Heart Failure 2012 of the European Society of Cardiology. Developed in collaboration with the Heart Failure Association (HFA) of the ESC. Eur J Heart Fail. 2012;14(8):803-69.

10. Yilmaz A, Gdynia HJ, Baccouche H, et al. Cardiac involvement in patients with Becker muscular dystrophy: new diagnostic and pathophysiological insights by a CMR approach. J Cardiovasc Magn Reson. 2008:10:50.

11. Quiat D, Olson EN. MicroRNAs in cardiovascular disease: from pathogenesis to prevention and treatment. J Clin Invest. 2013;123(1):11-8.

12. Zampetaki A, Mayr M. MicroRNAs in vascular and metabolic disease. Circ Res. 2012;110(3):508-22.

13. Chen $X, B a Y, M a L$, et al. Characterization of microRNAs in serum: a novel class of biomarkers for diagnosis of cancer and other diseases. Cell Res. 2008;18(10):997-1006

14. Gupta SK, Bang C, Thum T. Circulating microRNAs as biomarkers and potential paracrine mediators of cardiovascular disease. Circ Cardiovasc Genet. 2010;3(5):484-8.

15. Wang GK, Zhu JQ, Zhang JT, et al. Circulating microRNA: a novel potential biomarker for early diagnosis of acute myocardial infarction in humans. Eur Heart J. 2010:31(6):659-66.

16. Cerqueira MD, Weissman NJ, Dilsizian $\mathrm{V}$, et al. Standardized myocardial segmentation and nomenclature for tomographic imaging of the heart. A statement for healthcare professionals from the Cardiac Imaging Committee of the Council on Clinical Cardiology of the American Heart Association. Int J Cardiovasc Imaging. 2002;18(1):539-42.

17. Florian $A$, Rosch $S$, Bietenbeck $M$ et al Cardiac involvement in female Duchenne and Becker muscular dystrophy carriers in comparison to their first-degree male relatives: a comparative cardiovascular magnetic resonance study. Eur Heart J Cardiovasc Imaging. 2015;25.

18. Yilmaz A, Gdynia HJ, Mahrholdt H, Sechtem U. Cardiovascular magnetic resonance reveals similar damage to the heart of patients with Becker and limb-girdle muscular dystrophy but no cardiac symptoms. J Magn Reson Imaging. 2009;30(4):876-7.

19. Yan AT, Shayne AJ, Brown KA, et al. Characterization of the peri-infarct zone by contrast-enhanced cardiac magnetic resonance imaging is a powerful predictor of post-myocardial infarction mortality. Circulation. 2006;114(1):32-9.

20. Khairallah M, Khairallah R, Young ME, Dyck JR, Petrof BJ, Des RC. Metabolic and signaling alterations in dystrophin-deficient hearts precede overt cardiomyopathy. J Mol Cell Cardiol. 2007:43(2):119-29.

21. De AV, Serra F, Cogoni C, Vivarelli E, Monaco L, Naro F. beta1syntrophin modulation by miR-222 in mdx mice. PLoS One. 2010;5(8). doi:10.1371/journal.pone.0012098.

22. Ugander M, Oki AJ, Hsu LY, et al. Extracellular volume imaging by magnetic resonance imaging provides insights into overt and sub-clinical myocardial pathology. Eur Heart J. 2012:33(10):1268-78.

23. Florian A, Ludwig A, Engelen $M$, et al. Left ventricular systolic function and the pattern of late-gadolinium-enhancement independently and additively predict adverse cardiac events in muscular dystrophy patients. J Cardiovasc Magn Reson. 2014;16:81

24. Silva MC, Meira ZM, Gurgel GJ, et al. Myocardial delayed enhancement by magnetic resonance imaging in patients with muscular dystrophy. J Am Coll Cardiol. 2007:49(18):1874-9.

25. Florian A, Ludwig A, Ong $P$, et al. Cause of cardiac disease in a female carrier of Duchenne muscular dystrophy: myocarditis versus genetic cardiomyopathy without skeletal myopathy? Circulation. 2014;129(18):e482-4.

26. Gabriel RS, Kerr AJ, Sharma V, Zeng IS, Stewart RA. B-type natriuretic peptide and left ventricular dysfunction on exercise echocardiography in patients with chronic aortic regurgitation. Heart. 2008:94(7):897-902.

27. Sutton TM, Stewart RA, Gerber IL, et al. Plasma natriuretic peptide levels increase with symptoms and severity of mitral regurgitation. J Am Coll Cardiol. 2003;41(12):2280-7. 
28. Baccouche H, Mahrholdt H, Meinhardt G, et al. Diagnostic synergy of noninvasive cardiovascular magnetic resonance and invasive endomyocardial biopsy in troponin-positive patients without coronary artery disease. Eur Heart J. 2009:30(23):2869-79.

29. Hamm CW, Bassand JP, Agewall S, et al. ESC guidelines for the management of acute coronary syndromes in patients presenting without persistent STsegment elevation. The Task Force for the management of acute coronary syndromes (ACS) in patients presenting without persistent ST-segment elevation of the European Society of Cardiology (ESC). G Ital Cardiol (Rome). 2012;13(3):171-228

30. Eisenberg I, Eran A, Nishino I, et al. Distinctive patterns of microRNA expression in primary muscular disorders. Proc Natl Acad Sci U S A. 2007;104(43):17016-21.

31. Greco S, De SM, Colussi C, et al. Common micro-RNA signature in skeletal muscle damage and regeneration induced by Duchenne muscular dystrophy and acute ischemia. FASEB J. 2009;23(10):3335-46.

32. Hu J, Kong M, Ye Y, Hong S, Cheng L, Jiang L. Serum miR-206 and other muscle-specific microRNAs as non-invasive biomarkers for Duchenne muscular dystrophy. J Neurochem. 2014;129(5):877-83.

33. Jeanson-Leh L, Lameth J, Krimi S, et al. Serum profiling identifies novel muscle miRNA and cardiomyopathy-related miRNA biomarkers in Golden Retriever muscular dystrophy dogs and Duchenne muscular dystrophy patients. Am J Pathol. 2014;184(11):2885-98.

34. Orenes-Pinero E, Montoro-Garcia S, Patel JV, Valdes M, Marin F, Lip GY. Role of microRNAs in cardiac remodelling: new insights and future perspectives. Int J Cardiol. 2013:167(5):1651-9.

35. Roberts TC, Blomberg KE, McClorey G, et al. Expression analysis in multiple muscle groups and serum reveals complexity in the microRNA transcriptome of the mdx mouse with implications for therapy. Mol Ther Nucleic Acids. 2012;1, e39.

36. Thum T. Noncoding RNAs and myocardial fibrosis. Nat Rev Cardiol. 2014;11(11):655-63.

37. Vignier $N$, Amor F, Fogel $P$, et al. Distinctive serum miRNA profile in mouse models of striated muscular pathologies. PLoS ONE. 2013;8(2), e55281.

38. Wei C, Kim IK, Kumar S, et al. NF-kappaB mediated miR-26a regulation in cardiac fibrosis. J Cell Physiol. 2013;228(7):1433-42.

\section{Submit your next manuscript to BioMed Central and we will help you at every step:}

- We accept pre-submission inquiries

- Our selector tool helps you to find the most relevant journal

- We provide round the clock customer support

- Convenient online submission

- Thorough peer review

- Inclusion in PubMed and all major indexing services

- Maximum visibility for your research

Submit your manuscript at www.biomedcentral.com/submit 